\title{
The Era of Endovascular Treatment in Venous Disease: What Can We Do and Who Gets Benefit?
}

\author{
Hariadi Hariawan \\ Department of Cardiology and Vascular Medicine, Faculty of Medicine, Public Health and Nursing, Universitas \\ Gadjah Mada - Dr.Sardjito General Hospital, Yogyakarta, Indonesia
}

Corresponding author:

Hariadi Hariawan, MD, - email: hariadi_yk@ugm.ac.id

Department of Cardiology and Vascular Medicine, Faculty of Medicine, Public Health and Nursing, Universitas

Gadjah Mada - Dr.Sardjito Hospital

Jalan Farmako Sekip Utara,Yogyakarta,Indonesia 55281

\section{Introduction}

Venous system is very different and more complex than arterial system. To deliver blood against the forces of gravity, venous system rely on valve function and peripheral muscle pump. In acute venous disease, blood becomes stasis and leads to deep vein thrombosis. In chronic condition, clinical manifestation may vary from either venous obstruction or vascular incompetence. In general venous diseases are devided into venous thromboembolism and chronic venous insufficiency.

When it comes to venous diseases, clinicians, especially in Indonesia, tend to pay less attention and effort to make diagnosis, let alone to manage them. Veins are less understood affairs in cardiovascular yet fortunately, venology and endovascular field see rapid development in order to catch up with increasing incidence of venous disease.

Enormous burden have been placed in the shoulder of our society whether in economic, physical, and mental aspects. Lower extremities venous disease is one of the most prevalent cases. In certain circumstances it will develop into more life-threatening (acute pulmonary embolism) or limb-threatening venous ischemia (such as in phlegmasia cerulia dolens). While many questions remain more considerable than their answers, plentiful progress has been made in our acknoledgement of the disease and how or when to do the intervention. Many techniques have been invented and perfected to minimize bleeding and remove thrombus from the vein. Endovascular recanalization is now comparable against open surgery with much lower complications.

\section{Venous thromboembolism (VTE)}

A VTE is the third most common cardio-cerebro-vascular disease in the world after acute myocardial infarction and cerebrovascular disease (stroke). VTE consists of two main disease i.e. deep vein thrombosis (DVT) and pulmonary embolism (PE). ${ }^{1}$

Clinical manifestation of DVT of the legs are swelling or pitting edema, erythema, pain, and the presence of collateral superficial veins that would be well presented with duplex ultrasound as its main modality of diagnosis. $^{2}$

The most life-threatening complication of DVT is pulmonary embolism because of its significant case mortality rate. Historically, 
recommendation of treatment for DVT was to prevent pulmonary embolism using anticoagulant drugs.Initial therapy with means of parenteral anticoagulation drug (unfractionated heparin, low-molecular-weight heparin, or fondaparinux) bridging to long term anti-vitamin $\mathrm{K}$. Of course this practice was highly inconvenient because of drug interaction and troublesome monitoring. Thus, direct oral anticoagulation drugs such as rivaroxaban, apixaban, and dabigatran emerged to be drug of choices because its pharmacokinetics highly unaffected by diet with comparable efficacy in the prevention of recurrent VTE against anti-vitamin $\mathrm{K}$ drug. However, no antidote exists today to prevent serious bleeding caused by these drugs. ${ }^{3,4}$

\section{Post thrombotic syndrome (PTS) \\ Modernization of concept beyond pulmonary embolism} prevention has been suggested by contemporary studies. Post-thrombotic syndrome (PTS) is a gruesome condition that develop in approximately $40 \%$ patients with DVT. It can be felt until years after first diagnosed DVT. The symptoms of PTS are venous claudiation, fatigue, heaviness, and swelling that worsens with upright position and activities. VETO trials (Venous Thrombosis Outcomes) stated that PTS are the main predictors of patients quality of life 2 years after first diagnosed DVT. ${ }^{5}$

The PTS pathogenesis is still unclear. Initial inflammatory response to thrombosis affects the resolution, organization of thrombus, and injury of the vein wall. Loss of wall compliance, increase of wall thickness, and impaired vessel valvular function are the result of this mechanism. Vein thrombosis should be cleared from the deep venous system because inability to resolve it will block venous blood flow and do the damages of the venous valves as well as alters the adjacent vein wall. ${ }^{6}$

Rapid thrombus resolution and restoration of the flow in deep vein after thrombosis is the key to prevent PTS thus creating "open vein hypothesis". PTS predilection is in the proximal DVT patient who still had thrombus in the vein after 6 months. ${ }^{7}$ There are strong correlation between the size of residual thrombus after treatment with anticoagulant and the incidence of recurrent VTE, hence the development of PTS. Several studies proposed usage of systemic thrombolysis to clear the thrombus. Nevertheless, significant lysis happened in patient with nonobstructive thrombi instead of obstructive. This demonstrated that systemic administration cannot reach the target adequately. On the other hand, intrathrombus infusion of thrombolytic drugs has shown greater efficacy and maintained lower risk of bleeding. This principle gives rise to the current catheter-directed thrombolytic DVT therapy. ${ }^{8}$

\section{Catheter-directed thrombolysis (CDT)}

Systemic thrombolytic is not recommended for DVT therapy, only CDT is suggested. In DVT, endovascular treatment through catheter directed therapy is more acceptable than systemic administration, The reason is because of the increase of bleeding risk in systemic thrombolysis than using CDT. CDT delivers rapid and direct infusion of thrombolytic agent through fenestrated catheter so high local doses of drug even with reduced dose of systemic lytic agent within the 
thrombosed location, increase possibility to reduce clot and repair vessel patency and less major bleeding events. $^{9}$

A CDT delivers more concentrated drug into target site while reducing drug dosage. It was the first endovascular thrombolytic method to treat DVT. The best way to reach target vein is using vessels caudal to the thrombus and placing the tip of the catheter inside it. If the popliteal vein is patent, we can puncture it through this vein. However, if this vein is also occluded, we can use the fibularis vein or posterior tibial vein although superior skill is needed. Internal jugular vein or brachial vein using longer catheters and wires can also be considered. Catheters used in this practice are multi-side-hole catheter attached within the thrombus. Recombinant tissue plasminogen activator $(0.01 \mathrm{mg} / \mathrm{kg} / \mathrm{h}$ up to $1 \mathrm{mg} / \mathrm{h})$ is typically continued for 6-24 hours while patient is monitored for bleeding. Laboratory parameters such as fibrinogen, partial thromboplastin time, and fibrinogen level can be drawn every 6 hours. ${ }^{10}$

The onset of DVT is associated with successful treatment. CDT is probably to be successful in acute condition when onset is less than 14 days, while in subacute condition (between 14-28 days) is still beneficial in some studies. However, when the onset is more than 28 days, CDT successful is questionable. ${ }^{11}$

\section{Percutaneus transluminal angioplasty (PTA)}

Evaluation venography is perfomed to see the remaining thrombus and position of the catheter. If thrombus is still persist, percutaneus transluminal angioplasty (PTA) using balloon angioplasty can be done to increase surface area for thrombolysis and the infusion is continued. Stent placement are generally reserved for the large iliofemoral obstruction with good results. ${ }^{8}$ Routine use of inferior vena cava deemed unnecessary as pulmonary embolism complication of CDT is very low (1.3\%). ${ }^{12}$

With the advancement of technology in endovascular interventions, there are several techniques which could increase the efficacy of CDT such as combining percutaneous mechanical thrombectomy (PMT) with CDT. Pharmacomechanical catheter directed thrombolysis (PCDT) enhance intervensionist ability to perform large thrombus removal in patient with DVT. It can increase thrombus surface area, accelerate pharmacologic thrombolysis, reduce drug dose and duration, as well as reduce bleeding complications and risk of pulmonary embolism. ${ }^{13}$

\section{Angio Jet}

Angio Jet is a pulse-spray bolus dose of thrombolytic drug directly into the thrombus then the AngioJet catheter is used to aspirate the residual thrombus. The effectivenes of therapy can be reach in $80-90 \%$ of patients, half of it may be trated in single session. ${ }^{14}$

Catheter-directed thrombolysis is indicated in patient with extensive thrombosis with high risk of developing pulmonary embolism, proximal DVT (iliofemoral or femoral vein), threatened limb viability such as in phelgmasiacerulia dolens, good physicological reserve, recent onset of symptoms less than 14 days, with life expectancy more than 6 months, and absence of contraindications to thrombolysis. ${ }^{15}$ 
Thrombolysis for pulmonary embolism (PE)

A PE is the most fatal complication of DVT, together forms broader scope of venous thromboembolism (VTE). Intervention on pulmonary artery with local and directed thrombolysis has been an area of increasing interest. With systemic thrombolysis, the drug infused will be shunted towards unobstructerd pulmonary artery, thus limiting drug efficacy. On the contrary, catheter-directed thrombolysis promise more targeted therapy. Catheter available for thrombolysis were not design specifically for PE, instead for peripheral vasculature.

Combination CDT and thromboaspiration or mechanical thrombectomy is the treatment of choice for this condition. Other interventional option for patient with absolute contraindication to thrombolysis includes: thrombus fragmentation with pigtail or balloon catheter, rheolyticthrombectomy with hydrodynamic catheter device, suction thrombectomy with aspiration catheter and rotational thrombectomy.

ULTIMA (Ultrasound Accelerated Thrombolysis of Pulmonary Embolism) trial using ultrasoundassisted catheter-directed thrombolysis (USAT) randomized 59 patients with intermediate risk of PE to USAT versus heparin anticoagulation. The USAT arm used gradual targeted infusion of thrombolysis over 12 to 24 hour with alteplase dose of $1 \mathrm{mg} / \mathrm{h}$. Patients in the USAT arm was better in right ventricle/left ventricle ratio at 24 hour but the 90 day difference between USAT and heparin was statistically insignificant $(p=0.07)$ suggesting an early advantage to USAT but not in the long term. ${ }^{16}$ AngioJet used in DVT can also be the alternative to perform endovascular intervention in PE. Several case series reported that AngioJet procedure can induce bradycardia, hypotension, hypoxia, and hemodynamic collapse probably driven by release of vasoactive agents such as adenosine and bradykinin. ${ }^{17}$ Availabe data is still limited to support routine use of endovascular intervention in intermediate-high risk PE.

\section{Chronic venous insufficiency (CVI)}

A CVI is a pathological condition of the vein that implicated from prolonged stasis of venous blood flow. Venous hypertension gives rise to the chronic inflammation, edema, hyperpigmentation, eczema, lipodermatosclerosis, and ulcers. ${ }^{4}$ Varices of the lower limbs is a very occuring disease in adult with prevalence 5-15\% among men and 3$29 \%$ among women. Lifestyle changes such as weight loss, diet, regular exercise, and elevation of the feet in the afternoon are the initial conservative therapy. For smaller veins, sclerotherapy is the treatment of choice. It is one of the most regularly procedure performed with injection of chemical irritant to induce thrombopheblitis, occlusion, and vein fibrosis. Another procedure is phlebectomy whiche remove tributary veins through multiple skin incision. This performed with local anesthesia such as with lidocaine which after procedure completed patient used compression stocking for 1-2 weeks. ${ }^{18}$

\section{Endovascular laser therapy (EVLT)}

An EVLT is the latest and most recent breakthrough treatment for chronic venous insufficiency. It is generally considered after treatment 
with conservative therapy has failed. It works by ablating the innermost wall of the vein and induce direct heating effects. The lasers consist of continous and pulsed wave with the pulsed is more powerful than the first. The target for chromophore was the $810 \mathrm{~nm}$ and $940 \mathrm{~nm}$ laser. Treatment with EVLT started with color doppler USG to map areas of venous reflux then using guide wire to the target vein subsequently by the insertion of the laser energy tip. Once the laser is well positioned, it is activated and the laser fibre pulled back. Patients then use compression stockings to reduce complications such as tenderness and venous thromboembolism, are encouraged to walk immediately and returned to daily activities witihin days. ${ }^{19}$

\section{Conclusion}

Advancement in endovascular intervension of venous disease is very encouraging. Many different techniques are developed to match the prevalent nature of the disease. Catheter directed thrombolysis and it variances delivers more promising future for treatment of DVT although more scientific studies for pulmonary embolism is needed. Less drug dose is needed so less bleeding complication are found with catheter directed thrombolysis. An EVLT are contemporary management for CVI using laser heat to ablate dysfunctional and varicose veins. It gives less complication with favorable outcome compared to surgical management.

\section{References}

1. Raskob G.F., Angchaisuksiri P., Blanco A.N. 2014. Thrombosis: a major contributor to global disease burden. Arterioscler Thromb Vasc Biol, ;342363-71.

2. Di Nisio M., Van Es N.M, Buller H.R. 2017. Deep vein thrombosis and pulmonary embolism. Lancet, 388:3060-3073.

3. Bauersachs R., Berkowitz S.D., Brenner B., Buller H.R., Decousus H., Gallus A., et al. 2010. Oral rivaroxaban for symptomatic venous thromboembolism. $\mathrm{N}$ Engl J Med, 363:2499-2510.

4. Bradbury A., Evans C., Allan P., Lee A., Ruckley C.V., Fowkes F.G. 1999. What are the symptoms of varicose veins? Edinburgh vein study cross sectional population survey. BMJ, 318:353-356.

5. Kahn S.R., Shbaklo H., Lamping D.L., Holcroft C.A., Shrier I., Miron M.J.,, et al. 2008. Determinants of health-related quality of life during the 2 years following deep vein thrombosis. J Thromb Haemost, 6:1105-1112.

6. Deroo S., Deatrick K.B., Henke P.K. 2010. The vessel wall: $A$ forgotten player in post thrombotic syndrome. Thromb Haemost, 104:681-692.

7. Prandoni P., Frulla M., Sartor D., Concolato A., Girolami A. 2005. Vein abnormalities and the postthrombotic syndrome. J Thromb Haemost , 3:401-402.

8. Vedantham S., Thorpe P.E., Cardella J.F., Grassi C.J., Patel N.H., Ferral H., et al. 2006. Quality improvement guidelines for the treatment of lower extremity deep vein thrombosis with useof endovascular thrombus removal. J Vasc Interv Radiol, 17:435-447.

9. Lee K.A., Ramaswamy R.S. 2017. Interventional approaches to deep 
venous thrombosis. Curr Opin Cardiol, 32:679-686.

10. Semba C.P., Dake M.D. 1994. lliofemoral deep venous thrombosis: aggressive therapy with catheter-directed thrombolysis. Radiology, 191:487-494.

11. Sista A.K., Vedantham S., Kaufman J.A., Madoff D.C. 2015. Endovascular intervention for acute and chronic lower extremity deep venous disease: State of the art. Radiology, 276:315-352.

12. Mewissen M.W., Seabrook G.R., Meissner M.H., Cynamon J., Labropoulos N., Haughton S.H. 1999. Catheter-directed thrombolysis for lower extremity deep venous thrombosis: report of a national multicenter registry. Radiology, 211:39-49.

13. Kim H.S., Patra A., Paxton B.E., Khan J., Streiff M.B. 2006. Adjunctive percutaneous mechanical thrombectomy for lower-extremity deep vein thrombosis: clinical and economic outcomes. J Vasc Interv Radiol, 17:1099-1104.

14. Cynamon J., Stein E.G., Dym R.J., Jagust M.B., Binkert C.A., Baum R.A. 2006. A new method for aggressive management of deep vein thrombosis: retrospective study of the power pulse technique. $\mathrm{J}$ Vasc Interv Radiol, 17:1043-1049.

15. Patterson B.O., Hinchliffe R., Loftus I.M. 2010. Indications for catheter-directed thrombolisis in the management of acute proximal deep venous thrombosis, Arteriorscler Thromb Vasc Biol, 30:669-674.

16. Kucher N., Boekstegers P., Müller O.J., Kupatt C., Beyer-Westendorf J., Heitzer T., et al. 2014. Randomized, controlled trial of ultrasoundassisted catheterdirected thrombolysis for acute intermediate-risk pulmonary embolism. Circulation, 129:479486.

17. Zhu D.W. 2008. The potential mechanisms of bradyarrhythmias associated with AngioJet thrombectomy. J Invasive Cardiol, 20:2A-4A

18. Sadick N.S. 2006. Advances in the treatment of varicose veins: ambulatory phlebectomy, foam sclerotherapy, endovascular laser, and radiofrequency closure. Adv Dermatol, 22:139-156.

19. Darwood R.J., Gough M.J. 2009. Endovenous laser treatment for uncomplicated varicose veins. Phlebology, 24:50-61. 